PROCEEDINGS OF THE

AMERICAN MATHEMATICAL SOCIETY

Volume 137, Number 8, August 2009, Pages 2697-2707

S 0002-9939(09)09813-X

Article electronically published on February 4, 2009

\title{
RELAXED COMMUTANT LIFTING: EXISTENCE OF A UNIQUE SOLUTION
}

\author{
S. TER HORST
}

(Communicated by Marius Junge)

\begin{abstract}
In this paper we present necessary and sufficient conditions for the existence of a unique solution to the relaxed commutant lifting problem. The obtained conditions are more complicated than those for the classical commutant lifting setting, and earlier obtained sufficient conditions turn out not to be necessary conditions. It is also shown that these conditions simplify in certain special cases.
\end{abstract}

\section{INTRODUCTION}

In this paper we resolve an open problem concerning relaxed commutant lifting. The relaxed commutant lifting problem was introduced in [5], extending the classical commutant lifting theory of B. Sz.-Nagy and C. Foias 14 and its predecessor by D. Sarason [17. As mentioned in [5], relaxed commutant lifting covers the generalizations of the commutant lifting setting by Treil and Volberg [18] and the weighted version in 1 . In $[5$ it was shown that a particular (central) solution always exists. Representations of all solutions have been given in 17, 13, 8, 12. In the present paper we provide necessary and sufficient conditions under which the so-called central solution is the only solution to the relaxed commutant lifting problem.

The data for the relaxed commutant lifting problem is a set $\left\{A, T^{\prime}, U^{\prime}, R, Q\right\}$ consisting of five Hilbert space operators: the operator $A$ is a contraction mapping $\mathcal{H}$ into $\mathcal{H}^{\prime}$, the operator $U^{\prime}$ on $\mathcal{K}^{\prime}$ is a minimal isometric lifting of the contraction $T^{\prime}$ on $\mathcal{H}^{\prime}$; i.e., $U^{\prime}$ is an isometry on $\mathcal{K}^{\prime}$, with $\mathcal{H}^{\prime} \subset \mathcal{K}^{\prime}$ being cyclic for $U^{\prime}$ and $\Pi_{\mathcal{H}^{\prime}} U^{\prime}=T^{\prime} \Pi_{\mathcal{H}^{\prime}}$, and $R$ and $Q$ are operators from $\mathcal{H}_{0}$ to $\mathcal{H}$, satisfying

$$
T^{\prime} A R=A Q \quad \text { and } \quad R^{*} R \leq Q^{*} Q .
$$

Here we follow the convention that for a subspace $\mathcal{V}$ of a Hilbert space $\mathcal{W}$ the symbol $\Pi_{\mathcal{V}}$ stands for the orthogonal projection from $\mathcal{W}$ onto $\mathcal{V}$ viewed as an operator from $\mathcal{W}$ onto $\mathcal{V}$. Given this data set the relaxed commutant lifting problem is to find a (all) contraction(s) $B$ from $\mathcal{H}$ to $\mathcal{K}^{\prime}$ such that

$$
\Pi_{\mathcal{H}^{\prime}} B=A \quad \text { and } \quad U^{\prime} B R=B Q .
$$

A contraction $B$ from $\mathcal{H}$ into $\mathcal{K}^{\prime}$ that satisfies (2) is called a contractive interpolant for $\left\{A, T^{\prime}, U^{\prime}, R, Q\right\}$.

Received by the editors March 3, 2008, and, in revised form, October 20, 2008.

2000 Mathematics Subject Classification. Primary 47A20, 47A56, 47A57.

Key words and phrases. Commutant lifting, unique solution, linear fractional maps.

(C)2009 American Mathematical Society

Reverts to public domain 28 years from publication 
An essential role in the solution to the relaxed commutant lifting problem is played by the so-called underlying contraction $\omega$ (see [5]), which is defined by

$$
\omega: \mathcal{F}=\overline{D_{A} Q \mathcal{H}_{0}} \rightarrow\left[\begin{array}{c}
\mathcal{D}_{T^{\prime}} \\
\mathcal{D}_{A}
\end{array}\right], \quad \omega D_{A} Q=\left[\begin{array}{c}
D_{T^{\prime}} A R \\
D_{A} R
\end{array}\right]
$$

Here, as usual, given a contraction $N$, we write $D_{N}$ for the defect operator and $\mathcal{D}_{N}$ for the defect space of $N$; that is, $D_{N}$ is the positive square root of $I-N^{*} N$ and $\mathcal{D}_{N}$ is the closure of the range of $D_{N}$. The first component of $\omega$, mapping $\mathcal{F}$ into $\mathcal{D}_{T^{\prime}}$, is denoted by $\omega_{1}$, and the second component of $\omega$, mapping $\mathcal{F}$ into $\mathcal{D}_{A}$, by $\omega_{2}$.

The classical commutant lifting setting appears when $R$ is the identity operator on $\mathcal{H}$, and thus $\mathcal{H}_{0}=\mathcal{H}$, and $Q$ is an isometry. It is well known that in the classical commutant lifting setting there exists a unique contractive interpolant if and only if $\overline{\mathcal{D}_{A} Q \mathcal{H}_{0}}=\mathcal{D}_{A}$ or $\omega$ is unitary; cf. the first paragraph after Theorem VI.2.1 in [4.

For relaxed commutant lifting these conditions are sufficient but not necessary; see Example 2.2 below. The following theorem provides conditions for the relaxed commutant lifting setting that are both necessary and sufficient.

Theorem 0.1. There exists a unique contractive interpolant for the relaxed commutant lifting problem with data set $\left\{A, T^{\prime}, U^{\prime}, R, Q\right\}$ if and only if the underlying contraction $\omega$ satisfies one of the following conditions:

(i) $\overline{\mathcal{D}_{A} Q \mathcal{H}_{0}}=\mathcal{D}_{A}$,

(ii) the operator $\omega_{1}\left(\Pi_{\mathcal{F}} \omega_{2}\right)^{n}$ is a co-isometry for $n=0,1,2, \ldots$.

Condition (i) was already obtained as a sufficient condition; see Theorem 3.1 in 5]. Two other sufficient conditions were obtained earlier; both are covered by (ii): namely, the trivial condition $T^{\prime}$ is an isometry $\left(\mathcal{D}_{T^{\prime}}=\{0\}\right)$ (Proposition 6.6 in [7), and the condition that $\omega$ is a co-isometry (Section 4.1 in [11]). In [13] necessary and sufficient conditions are given for the case $R^{*} R=Q^{*} Q$. The equivalence of the conditions in [13] to those in Theorem 0.1 for the case $R^{*} R=Q^{*} Q$ is explained in Section 3

To see that this theorem covers the classical result it suffices to show that condition (ii) in the classical commutant lifting setting implies that $\omega$ is unitary; the sufficiency of the conditions follows from the last remark in the previous paragraph. Note that in the classical case $R^{*} R=Q^{*} Q$, which implies that the underlying contraction $\omega$ is an isometry; see [5]. Moreover, since $R$ is the identity operator, the range of $\omega_{2}$ is dense in $\mathcal{D}_{A}$. So if condition (ii) holds, then in particular $\omega_{1}$ is a co-isometry, and, as $\omega$ is an isometry and $\overline{\omega_{2} \mathcal{F}}=\mathcal{D}_{A}$, the contraction $\omega_{2}$ is also a co-isometry. This implies that $\omega$ is a co-isometry and thus unitary.

In order to prove Theorem 0.1 it is more convenient to work with the equivalent interpolation problem of [9]. To state the latter problem we first need some preliminaries. Let $\mathcal{U}$ and $\mathcal{Y}$ be Hilbert spaces. We write $\mathbf{H}^{2}(\mathcal{U}, \mathcal{Y})$ for the set of all operator-valued functions $H$ on the unit disc $\mathbb{D}$ of $\mathbb{C}$ whose values are operators from $\mathcal{U}$ into $\mathcal{Y}$ and such that the formula

$$
\left(\Gamma_{H} u\right)(\lambda)=H(\lambda) u \quad(u \in \mathcal{U}, \lambda \in \mathbb{D})
$$

defines an operator $\Gamma_{H}$ from $\mathcal{U}$ into the Hardy space $H^{2}(\mathcal{Y})$. The set $\mathbf{H}^{2}(\mathcal{U}, \mathcal{Y})$ is a Banach space under the norm $\|H\|=\left\|\Gamma_{H}\right\|$, where $\left\|\Gamma_{H}\right\|$ denotes the operator norm of $\Gamma_{H}$. We write $\mathbf{H}_{\text {ball }}^{2}(\mathcal{U}, \mathcal{Y})$ for the unit ball of $\mathbf{H}^{2}(\mathcal{U}, \mathcal{Y})$. 
With these definitions the interpolation problem of [9] can be formulated as follows: given a contraction

$$
\omega=\left[\begin{array}{l}
\omega_{1} \\
\omega_{2}
\end{array}\right]: \mathcal{F} \rightarrow\left[\begin{array}{l}
\mathcal{Y} \\
\mathcal{U}
\end{array}\right], \quad \text { where } \mathcal{F} \text { is a subspace of } \mathcal{U},
$$

describe a (all) function(s) $H$ in $\mathbf{H}_{\text {ball }}^{2}(\mathcal{U}, \mathcal{Y})$ such that

$$
\omega_{1}+\lambda H(\lambda) \omega_{2}=\left.H(\lambda)\right|_{\mathcal{F}} \quad(\lambda \in \mathbb{D}) .
$$

A function $H$ in $\mathbf{H}_{\text {ball }}^{2}(\mathcal{U}, \mathcal{Y})$ satisfying ([6) is called a solution to the $H^{2}$ interpolation problem defined by (the contraction) $\omega$, or just a solution when the contraction $\omega$ in question is clear from the context.

Notice that the underlying contraction $\omega$ in (3) is of the form (5). By making an appropriate choice for the data set, we find that each contraction $\omega$ of the form (5) appears as the underlying contraction for the corresponding relaxed commutant lifting problem. Moreover, given a data set $\left\{A, T^{\prime}, U^{\prime}, R, Q\right\}$ with underlying contraction $\omega$, there is a one-to-one map between the solutions $H$ of the $H^{2}$ interpolation problem defined by $\omega$ and the contractive interpolants $B$ for $\left\{A, T^{\prime}, U^{\prime}, R, Q\right\}$; this map is given by

$$
H \mapsto B=\left[\begin{array}{c}
A \\
\Gamma_{H} D_{A}
\end{array}\right],
$$

provided the minimal isometric lifting $U^{\prime}$ is the Sz.-Nagy-Schäffer isometric lifting on $\mathcal{H}^{\prime} \oplus H^{2}\left(\mathcal{D}_{T^{\prime}}\right)$, which we may assume without loss of generality. In this sense the two problems are equivalent; see [9] for details.

The following theorem is the analog of Theorem 0.1 for the $H^{2}$ interpolation setting.

Theorem 0.2. Let $\omega$ be a contraction of the form (5). Then there exists a unique solution to the $\mathrm{H}^{2}$ interpolation problem defined by $\omega$ if and only if one of the following conditions is met:

(i) $\mathcal{F}=\mathcal{U}$,

(ii) the operator $\omega_{1}\left(\Pi_{\mathcal{F}} \omega_{2}\right)^{n}$ is a co-isometry for $n=0,1,2, \ldots$.

We prove Theorem 0.2 in Section 2 Theorem 0.1 follows directly from Theorem 0.2 and the equivalence between the two problems, as explained above.

A solution to the $H^{2}$ interpolation problem defined by $\omega$ always exists. Indeed, it is not difficult to verify that the function $H_{c}$ on $\mathbb{D}$ given by

$$
H_{c}(\lambda)=\omega_{1} \Pi_{\mathcal{F}}\left(I_{\mathcal{U}}-\lambda \omega_{2} \Pi_{\mathcal{F}}\right)^{-1} \quad(\lambda \in \mathbb{D})
$$

is in $\mathbf{H}_{\text {ball }}^{2}(\mathcal{U}, \mathcal{Y})$ and satisfies (6) . This particular solution $H_{c}$ is referred to as the central solution and corresponds to the central solution for the relaxed commutant lifting problem obtained in 5 .

The operators $\omega_{1}\left(\Pi_{\mathcal{F}} \omega_{2}\right)^{n}$ in condition (ii) are closely related to the Taylor coefficients of the central solution $H_{c}$. Indeed, the $n^{\text {th }}$ Taylor coefficient of $H_{c}$ is equal to $\omega_{1} \Pi_{\mathcal{F}}\left(\omega_{2} \Pi_{\mathcal{F}}\right)^{n}=\omega_{1}\left(\Pi_{\mathcal{F}} \omega_{2}\right)^{n} \Pi_{\mathcal{F}}$, and thus condition (ii) holds if and only if the Taylor coefficients of $H_{c}$ are co-isometric. Now let $\Gamma_{H_{c}}$ be the contraction from $\mathcal{U}$ into $H^{2}(\mathcal{Y})$ associated with $H_{c}$ via (4). The fact that $\Gamma_{H_{c}}$ is contractive implies that $\Gamma_{H_{c}}$ is a co-isometry if and only if the Taylor coefficients of $H_{c}$ are co-isometries. In other words, condition (ii) in Theorem 0.2 is equivalent to:

(ii) ${ }^{\prime}$ the operator $\Gamma_{H_{c}}$ is a co-isometry.

In particular, we have the following corollary. 
Corollary 0.3. Let $\omega$ be a contraction of the form (5), and assume that $\mathcal{F} \neq \mathcal{U}$. Then $H_{c}$ is the only solution if and only if $\Gamma_{H_{c}}$ is a co-isometry.

This paper consists of three sections, not counting the present introduction. The first section contains preliminary material on a Redheffer representation of all solutions. In Section 2 we prove Theorem 0.2 , give an example and show how condition (ii) in Theorem 0.2 simplifies when additional conditions are imposed upon $\omega$. In the final section we revisit the original relaxed commutant lifting setting and specify Theorem 0.1 for some metric constrained interpolation problems.

We conclude the introduction with some words on the notation and terminology used in this paper. Throughout calligraphic letters denote Hilbert spaces. We write $\operatorname{dim}(\mathcal{U})$ for the dimension of $\mathcal{U}$. The Hilbert space direct sum of $\mathcal{U}$ and $\mathcal{Y}$ is denoted by

$$
\mathcal{U} \oplus \mathcal{Y} \quad \text { or } \quad\left[\begin{array}{l}
\mathcal{U} \\
\mathcal{Y}
\end{array}\right]
$$

By definition, a subspace is a closed linear manifold, and the closure of a linear subset $\mathscr{E}$ of $\mathcal{U}$ is denoted by $\overline{\mathscr{E}}$. If $\mathcal{M}$ is a subspace of $\mathcal{U}$, then $\mathcal{U} \ominus \mathcal{M}$ stands for the orthogonal complement of $\mathcal{M}$ in $\mathcal{U}$. We write $\mathcal{M}_{1} \vee \mathcal{M}_{2}$ for the closure of the linear span of the subspaces $\mathcal{M}_{1}$ and $\mathcal{M}_{2}$ of $\mathcal{U}$. The term operator stands for a bounded linear transformation acting between Hilbert spaces. We say that a contraction $A$ is a strict contraction if $\|A\|<1$ or, equivalently, when the defect operator $D_{A}$ is invertible. An operator $C$ from $\mathcal{U}$ into $\mathcal{Y}$ is said to be left invertible whenever there exists an operator $D$ from $\mathcal{Y}$ to $\mathcal{U}$ with $D C=I_{\mathcal{U}}$ (the identity operator on $\mathcal{U}$ ). In this case $D$ is called a left inverse of $C$. We say that a subspace $\mathcal{M}$ of $\mathcal{U}$ is cyclic for an operator $T$ on $\mathcal{U}$ if the smallest subspace of $\mathcal{U}$ that contains $T^{n} \mathcal{M}$ for each nonnegative integer $n$ is $\mathcal{U}$ itself. Finally, a Schur class function is an operator-valued function on the unit disc $\mathbb{D}$ whose values are contractions. The set of Schur class functions whose values are operators from $\mathcal{U}$ into $\mathcal{Y}$ is denoted by $\mathbf{S}(\mathcal{U}, \mathcal{Y})$ and is referred to as the Schur class associated with $\mathcal{U}$ and $\mathcal{Y}$.

\section{A RedhefFer RePRESEntation of ALL SOlutions}

Let $\omega$ be a contraction of the form (5). In this section we review some facts concerning the Redheffer representation of [11, Section 5.1] that describe all solutions to the $H^{2}$ interpolation problem defined by $\omega$.

Set $\mathcal{G}=\mathcal{U} \ominus \mathcal{F}$, and define operator-valued functions $\Phi_{1,1}, \Phi_{1,2}, \Phi_{2,1}$ and $\Phi_{2,2}$ on $\mathbb{D}$ by

$$
\begin{aligned}
\Phi_{1,1}(\lambda) & =\lambda \Pi_{\mathcal{G}}\left(I_{\mathcal{U}}-\lambda \omega_{2} \Pi_{\mathcal{F}}\right)^{-1} \Pi_{\mathcal{U}} D_{\omega^{*}}, \\
\Phi_{1,2}(\lambda) & =\Pi_{\mathcal{G}}\left(I_{\mathcal{U}}-\lambda \omega_{2} \Pi_{\mathcal{F}}\right)^{-1}, \\
\Phi_{2,1}(\lambda) & =\Pi_{\mathcal{Y}} D_{\omega^{*}}+\lambda \omega_{1} \Pi_{\mathcal{F}}\left(I_{\mathcal{U}}-\lambda \omega_{2} \Pi_{\mathcal{F}}\right)^{-1} \Pi_{\mathcal{U}} D_{\omega^{*}}, \\
\Phi_{2,2}(\lambda) & =\omega_{1} \Pi_{\mathcal{F}}\left(I_{\mathcal{U}}-\lambda \omega_{2} \Pi_{\mathcal{F}}\right)^{-1}
\end{aligned}
$$

for $\lambda \in \mathbb{D}$. Using the equivalence between relaxed commutant lifting and the $H^{2}$ interpolation problem defined by $\omega$, as described in [9], we find that Theorem 5.1.1 from [11] provides the following Redheffer representation of all solutions.

Theorem 1.1. Let $\omega$ be a contraction of the form (5), and let $\Phi_{1,1}, \Phi_{1,2}, \Phi_{2,1}$ and $\Phi_{2,2}$ be the functions given by (8). Then

$$
\Phi_{1,1} \in \mathbf{S}\left(\mathcal{D}_{\omega^{*}}, \mathcal{G}\right), \quad \Phi_{1,2} \in \mathbf{H}_{\text {ball }}^{2}(\mathcal{U}, \mathcal{G}), \quad \Phi_{2,1} \in \mathbf{S}\left(\mathcal{D}_{\omega^{*}}, \mathcal{Y}\right), \quad \Phi_{2,2} \in \mathbf{H}_{\text {ball }}^{2}(\mathcal{U}, \mathcal{Y}),
$$


and for any $V \in \mathbf{S}\left(\mathcal{G}, \mathcal{D}_{\omega^{*}}\right)$ the function $H$ given by

$$
H(\lambda)=\Phi_{2,2}(\lambda)+\Phi_{2,1}(\lambda) V(\lambda)\left(I-\Phi_{1,1}(\lambda) V(\lambda)\right)^{-1} \Phi_{1,2}(\lambda) \quad(\lambda \in \mathbb{D})
$$

is a solution to the $H^{2}$ interpolation problem defined by $\omega$. Moreover, all solutions are obtained in this way.

A representation of the form (9) is referred to as a Redheffer representation and the functions $\Phi_{1,1}, \Phi_{1,2}, \Phi_{2,1}$ and $\Phi_{2,2}$ as the corresponding Redheffer coefficients.

Since $\Phi_{1,1}$ is a Schur class function with $\Phi_{1,1}(0)=0$, it follows that $\Phi_{1,1}(\lambda)=$ $\lambda \Upsilon(\lambda)$ for some Schur class function $\Upsilon$ in $\mathbf{S}\left(\mathcal{D}_{\omega^{*}}, \mathcal{G}\right)$ and for each $\lambda \in \mathbb{D}$; see Lemma 2.4.1 in [11]. Thus $\Phi_{1,1}(\lambda)$ is a strict contraction for each $\lambda \in \mathbb{D}$, and the inverse in (9) is properly defined.

By taking $V \in \mathbf{S}\left(\mathcal{G}, \mathcal{D}_{\omega^{*}}\right)$ to be the zero function, we see that the function $\Phi_{2,2}$ is a solution. In fact, $\Phi_{2,2}$ is precisely the central solution $H_{c}$ in (7).

The map $V \mapsto H$ given by the Redheffer representation (9) is in general not one-to-one. It can happen that different $V$ 's yield the same solution $H$. However, this nonuniqueness in the representation can be made explicit; see [8, 11] for details.

In order to derive more properties of the Redheffer coefficients we require some results from linear system theory. The terminology used here corresponds to that in [4. A co-isometric system is a quadruple $\{A, B, C, D\}$, consisting of operators $A$ on a Hilbert space $\mathcal{X}, B$ from $\mathcal{V}$ to $\mathcal{X}, C$ from $\mathcal{X}$ to $\mathcal{W}$ and $D$ mapping $\mathcal{V}$ into $\mathcal{W}$ such that the operator matrix

$$
\left[\begin{array}{ll}
A & B \\
C & D
\end{array}\right]:\left[\begin{array}{l}
\mathcal{X} \\
\mathcal{V}
\end{array}\right] \rightarrow\left[\begin{array}{c}
\mathcal{X} \\
\mathcal{W}
\end{array}\right]
$$

is a co-isometry. Since $A$ is contractive, we can define functions $F$ and $W$ on $\mathbb{D}$ by

$$
F(\lambda)=D+\lambda C(I-\lambda A)^{-1} B \quad \text { and } \quad W(\lambda)=C(I-\lambda A)^{-1} \quad(\lambda \in \mathbb{D}) .
$$

We refer to $F$ and $W$ as the transfer function and observability function associated with $\{A, B, C, D\}$, respectively. From the fact that (10) is a co-isometry it follows that $F \in \mathbf{S}(\mathcal{V}, \mathcal{W})$ and $W \in \mathbf{H}_{\text {ball }}^{2}(\mathcal{X}, \mathcal{W})$. In particular, $F$ defines, in the usual way, a contractive multiplication operator $M_{F}$ from $H^{2}(\mathcal{V})$ to $H^{2}(\mathcal{W})$, and $W$ defines a contraction $\Gamma_{W}$ from $\mathcal{X}$ into $H^{2}(\mathcal{W})$ via (4).

Theorem 1.2. Let $\{A, B, C, D\}$ be a co-isometric system with transfer function $F \in \mathbf{S}(\mathcal{V}, \mathcal{W})$ and observability function $W \in \mathbf{H}_{\text {ball }}^{2}(\mathcal{X}, \mathcal{W})$. Then

$$
\left[\begin{array}{cc}
M_{F} & \Gamma_{W}
\end{array}\right]:\left[\begin{array}{c}
H^{2}(\mathcal{V}) \\
\mathcal{X}
\end{array}\right] \rightarrow H^{2}(\mathcal{W})
$$

is a co-isometry.

Proof. By Parrott's lemma [15] there exist operators $\widetilde{C}$ and $\widetilde{D}$ such that

$$
\left[\begin{array}{cc}
A & B \\
C_{1} & D_{1}
\end{array}\right]=\left[\begin{array}{c|c}
A & B \\
\hline C & D \\
\widetilde{C} & \widetilde{D}
\end{array}\right]:\left[\begin{array}{l}
\mathcal{X} \\
\mathcal{U}
\end{array}\right] \rightarrow\left[\begin{array}{c}
\mathcal{X} \\
\mathcal{Y} \\
\widetilde{\mathcal{Y}}
\end{array}\right]
$$

is unitary; that is, $\left\{A, B, C_{1}, D_{1}\right\}$ is a unitary system in the terminology of [4. The transfer function $F_{1}$ and observability function $W_{1}$ associated with $\left\{A, B, C_{1}, D_{1}\right\}$ then admit a decomposition of the form

$$
F_{1}(\lambda)=\left[\begin{array}{c}
F(\lambda) \\
\widetilde{F}(\lambda)
\end{array}\right] \quad \text { and } \quad W_{1}(\lambda)=\left[\begin{array}{c}
W(\lambda) \\
\widetilde{W}(\lambda)
\end{array}\right],
$$


with $\widetilde{F} \in \mathbf{S}(\mathcal{U}, \widetilde{\mathcal{Y}})$ and $\widetilde{W} \in \mathbf{H}_{\text {ball }}^{2}(\mathcal{X}, \widetilde{\mathcal{Y}})$, and it follows from Theorem III.10.4 in 4, identifying $\mathbf{H}^{2}(\mathcal{Y} \oplus \widetilde{\mathcal{Y}})$ with $\mathbf{H}^{2}(\mathcal{Y}) \oplus \mathbf{H}^{2}(\widetilde{\mathcal{Y}})$, that

$$
\left[\begin{array}{ll}
M_{F_{1}} & \Gamma_{W_{1}}
\end{array}\right]=\left[\begin{array}{ll}
M_{F} & \Gamma_{W} \\
M_{\widetilde{F}} & \Gamma_{\widetilde{W}}
\end{array}\right]
$$

is a co-isometry. Hence (11) is a co-isometry.

Since $\Phi_{1,1}$ and $\Phi_{2,1}$ are Schur class functions, they define contractive multiplication operators $M_{\Phi_{1,1}}$ from $H^{2}\left(\mathcal{D}_{\omega^{*}}\right)$ into $H^{2}(\mathcal{G})$ and $M_{\Phi_{2,1}}$ from $H^{2}\left(\mathcal{D}_{\omega^{*}}\right)$ into $H^{2}(\mathcal{Y})$, respectively. Moreover, Theorem 1.1 implies that we can define contractions $\Gamma_{\Phi_{1,2}}$ from $\mathcal{U}$ into $H^{2}(\mathcal{G})$ and $\Gamma_{\Phi_{2,2}}$ from $\mathcal{U}$ into $H^{2}(\mathcal{Y})$ associated with $\Phi_{1,2}$, respectively $\Phi_{2,2}$, via (4).

Now let the system $\{A, B, C, D\}$ be given by

$$
\left[\begin{array}{cc}
A & B \\
C & D
\end{array}\right]=\left[\begin{array}{c|c}
\omega_{1} \Pi_{\mathcal{F}} & \Pi_{\mathcal{U}} D_{\omega^{*}} \\
\hline \omega_{2} \Pi_{\mathcal{F}} & \Pi_{\mathcal{Y}} D_{\omega^{*}} \\
\Pi_{\mathcal{G}} & 0
\end{array}\right]:\left[\begin{array}{c}
\mathcal{U} \\
\mathcal{D}_{\omega^{*}}
\end{array}\right] \rightarrow\left[\begin{array}{c}
\mathcal{U} \\
\mathcal{Y} \\
\mathcal{G}
\end{array}\right]
$$

It follows from Douglas' factorization lemma [2] that $\{A, B, C, D\}$ is a co-isometric system. Applying Theorem 1.2 to this system and using that we can identify $H^{2}(\mathcal{Y} \oplus \mathcal{G})$ with $H^{2}(\mathcal{Y}) \oplus H^{2}(\mathcal{G})$, we obtain the following result.

Theorem 1.3. Let $\omega$ be a contraction of the form (5), let $M_{\Phi_{1,1}}$ and $M_{\Phi_{2,1}}$ be the multiplication operators defined by $\Phi_{1,1}$ and $\Phi_{2,1}$ in (8), respectively, and let $\Gamma_{\Phi_{1,2}}$ and $\Gamma_{\Phi_{2,2}}$ be the operators associated with $\Phi_{1,2}$ and $\Phi_{2,2}$ in (8) via (44), respectively. Then the operator

$$
\left[\begin{array}{ll}
M_{\Phi_{1,1}} & \Gamma_{\Phi_{1,2}} \\
M_{\Phi_{2,1}} & \Gamma_{\Phi_{2,2}}
\end{array}\right]:\left[\begin{array}{c}
H^{2}\left(\mathcal{D}_{\omega^{*}}\right) \\
\mathcal{U}
\end{array}\right] \rightarrow\left[\begin{array}{l}
H^{2}(\mathcal{G}) \\
H^{2}(\mathcal{Y})
\end{array}\right]
$$

is a co-isometry.

From the fact that the coefficient matrix (12) is a co-isometry we immediately obtain the following corollary.

Corollary 1.4. Let $\omega$ be a contraction of the form (5), and let $\Phi_{2,1}$ and $\Phi_{2,2}$ be the functions given by (8). Then $\Gamma_{\Phi_{2,2}}$ is a co-isometry if and only if $\Phi_{2,1}(\lambda)=0$ for each $\lambda \in \mathbb{D}$.

\section{Existence of A Unique SOlution}

In this section we prove Theorem 0.2 and give an example that shows that the earlier obtained sufficient condition " $\mathcal{F}=\mathcal{U}, \mathcal{Y}=\{0\}$ or $\omega$ is a co-isometry" is not a necessary condition. We conclude the section with a proposition that shows how condition (ii) in Theorem 0.2 simplifies in two special cases.

It will be convenient to first prove the following lemma.

Lemma 2.1. Let $A \in \mathbf{S}(\mathcal{W}, \mathcal{V})$, and let $B$ and $C$ be operator-valued analytic functions on $\mathbb{D}$ whose values are operators from $\mathcal{U}$ to $\mathcal{V}$ and from $\mathcal{W}$ to $\mathcal{Y}$, respectively. Then

$$
C(\lambda) V\left(I_{\mathcal{V}}-A(\lambda) V\right)^{-1} B(\lambda)=0 \quad(\lambda \in \mathbb{D})
$$

for any strict contraction $V$ from $\mathcal{V}$ to $\mathcal{W}$ if and only if

$$
B(\lambda)=0 \quad(\lambda \in \mathbb{D}) \quad \text { or } \quad C(\lambda)=0 \quad(\lambda \in \mathbb{D}) .
$$


Proof. The sufficiency of (14) is obvious. So assume that (13) holds for any strict contraction $V$ from $\mathcal{V}$ to $\mathcal{W}$, and assume in addition that $B$ is not the zero function. Then there exist a $u \in \mathcal{U}$ and $\lambda_{0} \in \mathbb{D}$ such that $u \neq 0$ and $B\left(\lambda_{0}\right) u \neq 0$. By the continuity of $B$ there exists a $\rho>0$ such that $B(\lambda) u \neq 0$ for all $\lambda$ in the open disc $\Delta\left(\lambda_{0}, \rho\right)=\left\{\lambda \in \mathbb{D}|| \lambda-\lambda_{0} \mid<\rho\right\} \subset \mathbb{D}$.

Now fix a $\lambda \in \Delta\left(\lambda_{0}, \rho\right)$ and an $h \in \mathcal{W}$. Let $\delta>0$ be small enough so that

$$
\|B(\lambda) u+\delta A(\lambda) h\|>\delta\|h\| .
$$

Then we can define a strict contraction $V$ from $\mathcal{V}$ to $\mathcal{W}$ by $V(B(\lambda) u+\delta A(\lambda) h)=\delta h$ and $V k=0$ for each $k \in \mathcal{G}$ perpendicular to the vector $B(\lambda) u+\delta A(\lambda) h$. So

$$
\delta(I-V A(\lambda)) h=V B(\lambda) u,
$$

and thus

$$
V(I-A(\lambda) V)^{-1} B(\lambda) u=(I-V A(\lambda))^{-1} V B(\lambda) u=\delta h .
$$

Then

$$
C(\lambda) h=\delta^{-1} C(\lambda) V(I-A(\lambda) V)^{-1} B(\lambda) u=0 .
$$

Since $h$ is an arbitrary vector in $\mathcal{W}$, we have $C(\lambda)=0$ for each $\lambda$ in the open disc $\Delta\left(\lambda_{0}, \rho\right)$. But $C$ is analytic on $\mathbb{D}$; thus $C(\lambda)=0$ for each $\lambda \in \mathbb{D}$.

Proof of Theorem 0.2. If there is just one solution, this unique solution must be the central solution $H_{c}$ in (7) (or $\Phi_{2.2}$ in the Redheffer representation (9)). It thus follows that there is a unique solution if and only if the second summand in (9) is the zero function for each $V \in \mathbf{S}\left(\mathcal{G}, \mathcal{D}_{\omega^{*}}\right)$, that is, if and only if

$$
\Phi_{2,1}(\lambda) V(\lambda)\left(I-\Phi_{1,1}(\lambda) V(\lambda)\right)^{-1} \Phi_{1,2}(\lambda)=0 \quad\left(\lambda \in \mathbb{D}, V \in \mathbf{S}\left(\mathcal{G}, \mathcal{D}_{\omega^{*}}\right)\right) .
$$

It follows from Lemma 2.1 that (15) is equivalent to

$$
\Phi_{1,2}(\lambda)=0 \quad(\lambda \in \mathbb{D}) \quad \text { or } \quad \Phi_{2,1}(\lambda)=0 \quad(\lambda \in \mathbb{D}) .
$$

The definition of $\Phi_{1,2}$ shows that $\Phi_{1,2}(\lambda)=0$ for all $\lambda \in \mathbb{D}$ if and only if $\mathcal{G}=\{0\}$, or equivalently, $\mathcal{F}=\mathcal{U}$. Next, from Corollary 1.4 and the remark in the paragraph preceding Corollary 0.3 we see that the second condition in (16) is equivalent to condition (ii) in Theorem 0.2 . This completes the proof.

Example 2.2. Let $\mathcal{Y}=\mathbb{C}$ and $\mathcal{U}=\ell_{+}^{2}$; that is, $\mathcal{U}$ is the Hilbert space of square summable unilateral sequences of complex numbers. Set $\mathcal{F}=S \ell_{+}^{2}$, where $S$ denotes the unilateral forward shift on $\ell_{+}^{2}$. Now define

$$
\omega=\left[\frac{\omega_{1}}{\omega_{2}}\right]=\left[\begin{array}{cccc}
1 & 0 & 0 & \cdots \\
\hline 0 & 0 & 0 & \cdots \\
0 & 1 & 0 & \cdots \\
0 & 0 & 1 & \ddots \\
\vdots & \vdots & \ddots & \ddots
\end{array}\right]: \mathcal{F} \rightarrow\left[\frac{\mathbb{C}}{\ell_{+}^{2}}\right] .
$$

Then $\mathcal{F} \neq \ell_{+}^{2}=\mathcal{U}, \omega$ is not a co-isometry and $\mathcal{Y}=\mathbb{C} \neq\{0\}$. However, we do have

$$
\omega_{1}\left(\Pi_{\mathcal{F}} \omega_{2}\right)^{n}=\left[\begin{array}{lllllll}
0 & \cdots & 0 & 1 & 0 & 0 & \cdots
\end{array}\right]: \mathcal{F} \rightarrow \mathbb{C} \text { for } n=0,1,2, \ldots,
$$

where the number 1 is situated in the $n^{\text {th }}$ position. In particular, $\omega_{1}\left(\Pi_{\mathcal{F}} \omega_{2}\right)^{n}$ is a co-isometry for each nonnegative integer $n$. So by Theorem 0.2 the central solution 
$H_{c}$ is the only solution to the $H^{2}$ interpolation problem defined by $\omega$. It is now easy to see that $H_{c}$ is given by

$$
H_{c}(\lambda)=\left[\begin{array}{llllll}
0 & 1 & \lambda & \lambda^{2} & \lambda^{3} & \ldots
\end{array}\right] \quad(\lambda \in \mathbb{D}) .
$$

Proposition 2.3. Let $\omega$ be a contraction of the form (5). If either $\omega_{1}$ is a strict contraction or $\operatorname{dim}(\mathcal{U})<\infty$, then condition (ii) in Theorem 0.2 is equivalent to $\mathcal{Y}=\{0\}$

Proof. Clearly $\mathcal{Y}=\{0\}$ implies that $\omega_{1}\left(\Pi_{\mathcal{F}} \omega_{2}\right)^{n}$ from $\mathcal{F}$ into $\{0\}$ is a co-isometry. If $\omega_{1}$ is a strict contraction, then $\omega_{1}$ can only be a co-isometry if $\mathcal{Y}=\{0\}$. Hence in that case condition (ii) in Theorem 0.2 reduces to $\mathcal{Y}=\{0\}$.

Next, assume that $\operatorname{dim}(\mathcal{U})<\infty$. We already proved that $\mathcal{Y}=\{0\}$ implies condition (ii) in Theorem 0.2 . Now assume that condition (ii) in Theorem 0.2 holds. Set $\mathcal{F}_{n}=\overline{\left(\omega_{2}^{*} \Pi_{\mathcal{F}}^{*}\right)^{n} \omega_{1}^{*} \mathcal{Y}}$ for $n=0,1,2, \ldots$. Since $\omega_{1}\left(\Pi_{\mathcal{F}} \omega_{2}\right)^{n}$ is a co-isometry, $\operatorname{dim}\left(\mathcal{F}_{n}\right)=\operatorname{dim}(\mathcal{Y})$. Moreover, $\omega_{1}$ maps $\mathcal{F}_{0}$ isometrically onto $\mathcal{Y}$ and $\omega_{2}$ maps $\mathcal{F}_{n+1}$ isometrically onto $\mathcal{F}_{n}$. Hence the subspaces $\mathcal{F}_{0}, \mathcal{F}_{1}, \ldots$ of $\mathcal{F}$ are mutually orthogonal and all of the same dimension as $\mathcal{Y}$. This is in contradiction with the fact that $\mathcal{F} \subset \mathcal{U}$ is finite dimensional, unless $\mathcal{Y}=\{0\}$.

\section{Relaxed COMMUTANT LIFTING}

In this section we return to the relaxed commutant lifting setting. We consider two special cases and explain the relation of Theorem 0.1 with a result of [13].

The suboptimal case. For most of the metric constrained interpolation problems that fit into the commutant lifting setting there is an interesting special case known as the suboptimal case; cf. [6] for the Nehari problem. On the level of commutant lifting this corresponds to the operator $A$ being a strict contraction, in which case a more explicit description of all solutions can be obtained (Section XIV.7 in 3 , Section VI.6 in [4). To achieve a similar result in the relaxed commutant lifting setting it is in addition assumed that $R$ has a left inverse; see [12. In that case we have the following result.

Corollary 3.1. Let $\left\{A, T^{\prime}, U^{\prime}, R, Q\right\}$ be a data set with $A$ a strict contraction and $R$ left invertible. Then there exists a unique contractive interpolant for $\left\{A, T^{\prime}, U^{\prime}, R\right.$, $Q\}$ if and only if $\overline{Q \mathcal{H}_{0}}=\mathcal{H}$ or $T^{\prime}$ is an isometry.

Proof. Note that $A$ being a strict contraction corresponds to $D_{A}$ being invertible on $\mathcal{H}$. The second condition in (11) implies that $Q$ has a left inverse whenever $R$ has a left inverse. So the conditions on the data set imply that both $D_{A} Q$ and $D_{A} R$ have left inverses, and thus

$$
\omega_{2}=\left.D_{A} R\left(Q^{*} D_{A}^{2} Q\right)^{-1} Q^{*} D_{A}\right|_{\mathcal{F}}
$$

has a left inverse as well. The latter implies that $\omega_{1}$ is a strict contraction, and thus, by Proposition 2.3 condition (ii) in Theorem 0.1 reduces to $\mathcal{D}_{T^{\prime}}=\{0\}$; that is, $T^{\prime}$ is an isometry. Since $D_{A}$ is invertible, it follows that condition (i) in Theorem 0.1 is equivalent to $\overline{Q \mathcal{H}_{0}}=\mathcal{H}$. 
Relaxations of metric constrained interpolation problems. As a motivation for the relaxed commutant lifting problem, in 5. a number of classical metric constrained interpolation problems, including Nevanlinna-Pick and Sarason interpolation, were provided with a relaxed version. The common ingredient in these problems is that in the relaxed commutant lifting setting $R$ and $Q$ are of the form

$$
R=\left[\begin{array}{cccc}
I_{\mathcal{V}} & 0 & \cdots & 0 \\
0 & I_{\mathcal{V}} & \ddots & \vdots \\
\vdots & \ddots & \ddots & 0 \\
0 & \cdots & 0 & I_{\mathcal{V}} \\
0 & \cdots & 0 & 0
\end{array}\right] \text { and } Q=\left[\begin{array}{cccc}
0 & 0 & \cdots & 0 \\
I_{\mathcal{V}} & 0 & \cdots & 0 \\
0 & \ddots & \ddots & \vdots \\
\vdots & \ddots & I_{\mathcal{V}} & 0 \\
0 & \cdots & 0 & I_{\mathcal{V}}
\end{array}\right] \text {, }
$$

where $\mathcal{V}$ is a given Hilbert space. In particular, if $\operatorname{dim}(\mathcal{V})<\infty$, then $\operatorname{dim}(\mathcal{H})=$ $\operatorname{dim}\left(\mathcal{V}^{n}\right)=\operatorname{dim}(\mathcal{V})^{n}<\infty$, and thus $\operatorname{dim}\left(\mathcal{D}_{A}\right)<\infty$. So it follows from Proposition 2.3 that in this case, condition (ii) of Theorem 0.1 reduces to $\mathcal{D}_{T^{\prime}}=\{0\}$; i.e., $T^{\prime}$ is an isometry.

A typical result for the relaxed interpolation problems of [5] is that in the special case $\mathcal{V}=\mathbb{C}$ there exists a unique contractive interpolant whenever the operator $A$ has norm one. We show here that this is the case for each relaxed commutant lifting problem with $R$ and $Q$ of the form (17) and $\mathcal{V}=\mathbb{C}$. First we prove the following lemma.

Lemma 3.2. Let $\left\{A, T^{\prime}, U^{\prime}, R, Q\right\}$ be a data set for the relaxed commutant lifting problem, and set $\mathcal{G}=\mathcal{D}_{A} \ominus \mathcal{F}$. Then $\overline{D_{A} \mathcal{G}}$ is perpendicular to both $Q \mathcal{H}_{0}$ and $\operatorname{Ker} D_{A}$. In particular, $\mathcal{F}=\mathcal{D}_{A}$ whenever $\overline{Q \mathcal{H}_{0}} \vee \operatorname{Ker} D_{A}=\mathcal{H}$.

Proof. Let $g \in \mathcal{G}=\operatorname{Ker} Q^{*} D_{A} \subset \mathcal{D}_{A}$. Then $D_{A} g \in \operatorname{Ker} Q^{*}$, or equivalently, $D_{A} g$ is perpendicular to $Q \mathcal{H}_{0}$. Since $D_{A}$ is a selfadjoint operator, it follows directly that $D_{A} g$ is perpendicular to $\operatorname{Ker} D_{A}$. The last statement of Lemma 3.2 holds because $D_{A}$ restricted to $\mathcal{G}$ is one-to-one.

Corollary 3.3. Let $\left\{A, T^{\prime}, U^{\prime}, R, Q\right\}$ be a data set for the relaxed commutant lifting problem with $R$ and $Q$ given by (17), $\mathcal{V}=\mathbb{C}$ and $\mathcal{D}_{T^{\prime}} \neq\{0\}$. Then there exists a unique contractive interpolant if and only if $A$ has norm one.

Proof. In case the norm of $A$ is less than one, we are in the suboptimal case described above, while $\operatorname{ker} Q^{*} \neq\{0\}$ and $\mathcal{D}_{T^{\prime}} \neq\{0\}$. Thus there is more than one contractive interpolant.

Now assume that $\|A\|=1$. Since $\mathcal{H}=\mathbb{C}^{n}$ is finite dimensional, this implies that $A$ has a norm-attaining vector, i.e., $\operatorname{Ker} D_{A} \neq\{0\}$. Note that $\operatorname{Ker} Q^{*}=\mathbb{C} \oplus\{0\}^{n-1}$. So according to Lemma 3.2 it suffices to show that there exists a norm-attaining vector $h=\left(h_{1}, \ldots, h_{n}\right) \in \mathbb{C}^{n}$ for $A$ with $h_{1} \neq 0$.

Let $h=\left(h_{1}, \ldots, h_{n}\right) \neq 0$ be a norm-attaining vector, but assume that $h_{1}=0$. Then $h=Q k$, with $k=\left(h_{2}, \ldots, h_{n}\right) \in \mathbb{C}^{n-1}$. We have

$$
\|R k\|=\|h\|=\|A h\|=\|A Q k\|=\left\|T^{\prime} A R k\right\| \leq\|A R k\| \leq\|R k\| .
$$

Thus $R k=\left(h_{2}, \ldots, h_{n}, 0\right)$ is also a norm-attaining vector. It may happen that $h_{2}=0$, in which case we just repeat the above procedure. After at most $n-1$ times we arrive at a norm-attaining vector $h=\left(h_{1}, \ldots, h_{n}\right)$ with $h_{1} \neq 0$. 
The Li-Timotin criterion. In [13, Proposition 6.2] for the case that $R^{*} R=Q^{*} Q$, a seemingly different necessary and sufficient criterion for the existence of a unique solution is obtained. Let $\left\{A, T^{\prime}, U^{\prime}, R, Q\right\}$ be a data set and assume that $R^{*} R=$ $Q^{*} Q$ and $T^{\prime}$ is a co-isometry. The latter assumption can be made without loss of generality; the first implies that the underlying contraction $\omega$ is an isometry. Let $V$ be the partial isometry given by

$$
V=\left[\begin{array}{cc}
T^{\prime} & \Pi_{\mathcal{D}_{T^{\prime}}}^{*} \omega_{1} \Pi_{\mathcal{F}} \\
0 & \omega_{2} \Pi_{\mathcal{F}}
\end{array}\right] \text { on }\left[\begin{array}{c}
\mathcal{H}^{\prime} \\
\mathcal{D}_{A}
\end{array}\right]
$$

(In 13] $V$ corresponds to an operator called $\omega$, but we do not use that notation here for obvious reasons.) Proposition 6.2 in 13 says that a unique solution exists if and only if one of two conditions is met. The second condition corresponds to condition (i) in Theorem 0.1 (cf. the proof of Corollary 4.2 in [13]). The first condition, after some translation, can be rephrased as:

(ii) ${ }^{\prime \prime} V^{* n} \mathcal{H}^{\prime} \subset \mathcal{H}^{\prime} \vee \omega \mathcal{F}=\left\{h+\omega f \mid h \in \mathcal{H}^{\prime}, f \in \mathcal{F}\right\}$ for $n=0,1,2, \ldots$

Writing out $V^{* n}$ we obtain that $\left.V^{* n}\right|_{\mathcal{D}_{T^{\prime}}}=\left(\Pi_{\mathcal{F}}^{*} \omega_{2}^{*}\right)^{n-1} \Pi_{\mathcal{F}}^{*} \omega_{1}^{*}$. Moreover, it also follows that (ii)" is equivalent to:

$$
\mathcal{D}_{T^{\prime}} \subset \omega \mathcal{F} \quad \text { and } \quad\left(\Pi_{\mathcal{F}}^{*} \omega_{2}^{*}\right)^{n} \Pi_{\mathcal{F}}^{*} \omega_{1}^{*} \mathcal{D}_{T^{\prime}} \subset \omega \mathcal{F} \text { for } n=0,1,2, \ldots
$$

Since $\omega$ is an isometry, and thus one-to-one, the first inclusion just means that $\omega_{1}$ is a co-isometry. It also implies that $\omega \mathcal{F}=\mathcal{D}_{T^{\prime}} \oplus \widetilde{\mathcal{U}}$ with $\widetilde{\mathcal{U}}=\omega_{2} \mathcal{F}$. The additional inclusions are thus equivalent to $\left(\Pi_{\mathcal{F}}^{*} \omega_{2}^{*}\right)^{n} \Pi_{\mathcal{F}}^{*} \omega_{1}^{*} \mathcal{D}_{T^{\prime}} \subset \widetilde{\mathcal{U}}$ for $n=0,1,2, \ldots$ Now, using that $\left.\Pi_{\mathcal{F}}^{*} \omega_{2}^{*}\right|_{\tilde{\mathcal{U}}}$ is an isometry, again because $\omega$ is an isometry, we find, recursively, that the second part of (18) is equivalent to $\left(\Pi_{\mathcal{F}}^{*} \omega_{2}^{*}\right)^{n} \Pi_{\mathcal{F}}^{*} \omega_{1}^{*}$ being isometric for $n=1,2, \ldots$ Thus (ii) ${ }^{\prime \prime}$ is equivalent to condition (ii) in Theorem 0.1 .

\section{ACKNOWLEDGEMENT}

The author thanks Prof. M.A. Kaashoek and Prof. A.E. Frazho for their useful comments and suggestions.

\section{REFERENCES}

[1] A. Biswas, C. Foias and A. E. Frazho, Weighted commutant lifting, Acta Sci. Math. (Szeged) 65 (1999), 657-686. MR.1737279 (2001b:47027)

[2] R.G. Douglas, On majorization, factorization, and range inclusion of operators on Hilbert space, Proc. Amer. Math. Soc. 17 (1966), 413-415. MR0203464 (34:3315)

[3] C. Foias and A. E. Frazho, The Commutant Lifting Approach to Interpolation Problems, Operator Theory 44, Birkhäuser-Verlag, Basel, 1990. MR,1120546 (92k:47033)

[4] C. Foias, A. E. Frazho, I. Gohberg and M.A. Kaashoek, Metric Constrained Interpolation, Commutant Lifting and Systems, Operator Theory 100, Birkhäuser-Verlag, Basel, 1998. MR1635831 (99i:47027)

[5] C. Foias, A.E. Frazho, and M.A. Kaashoek, Relaxation of metric constrained interpolation and a new lifting theorem, Integral Equations Operator Theory 42 (2002), 253-310. MR1875186 (2002j:47009)

[6] B.A. Francis, A Course in $H_{\infty}$ Control Theory, Lecture Notes in Control and Information Sciences 88, Springer-Verlag, Berlin, 1987. MR932459 (89i:93002)

[7] A.E. Frazho, S. ter Horst, and M.A. Kaashoek, Coupling and relaxed commutant lifting, Integral Equations Operator Theory 54 (2006), 33-67. MR2195229 (2007c:47007)

[8] A.E. Frazho, S. ter Horst, and M.A. Kaashoek, All solutions to the relaxed commutant lifting problem, Acta Sci. Math. (Szeged) 72 (2006), 299-318. MR2249492(2008d:47020) 
[9] A.E. Frazho, S. ter Horst, and M.A. Kaashoek, Relaxed commutant lifting: An equivalent version and a new application, Operator Theory 187, pp. 157-168, Birkhäuser-Verlag, Basel, 2008.

[10] K. Glover, All optimal Hankel-norm approximations of linear multivariable systems and their $L^{\infty}$-error bounds, Internat. J. Control 39 (1984), 1115-1193. MR.748558 (86a:93029)

[11] S. ter Horst, Relaxed commutant lifting and Nehari interpolation, Ph.D. Thesis, Vrije Universiteit, Amsterdam, 2007, available online: www.darenet.nl.

[12] S. ter Horst, Relaxed commutant lifting and a relaxed Nehari problem: Redheffer state space formulas, Math. Nachr., to appear.

[13] W.S. Li and D. Timotin, The relaxed intertwining lifting in the coupling approach, Integral Equations Operator Theory 54 (2006), 97-111. MR2195232(2006i:47021)

[14] B. Sz.-Nagy and C. Foias, Dilation des commutants d'opérateurs, C. R. Acad. Sci. Paris, Série A 266 (1968), 493-495. MR0236755 (38:5049)

[15] S. Parrott, On a quotient norm and the Sz.-Nagy-Foiaş lifting theorem, J. Funct. Anal. 30 (1978), 311-328. MR518338 (81h:47006)

[16] D.R. Pik, Block lower triangular operators and optimal contractive systems, Ph.D. Thesis, Vrije Universiteit, Amsterdam, 1999.

[17] D. Sarason, Generalized interpolation in $H^{\infty}$, Trans. Amer. Math. Soc. 127 (1967), 179-203. MR0208383(34:8193)

[18] S. Treil and A. Volberg, A fixed point approach to Nehari's problem and its applications, in: Toeplitz Operators and Related Topics (Santa Cruz, CA, 1992), The Harold Widom Anniversary Volume, Operator Theory 71, pp. 165-186, Birkhäuser-Verlag, Basel, 1994. MR1300219 (95i:47026)

Department of Mathematics, Virginia Tech, Blacksburg, Virginia 24061-0123

E-mail address: terhorst@math.vt.edu 